\title{
Posterior muscle-fascial reconstruction and knotless urethro-neo bladder anastomosis during robot-assisted radical cystectomy: Description of the technique and its impact on urinary continence
}

\author{
Federico Mineo Bianchi ${ }^{1,2}$, Daniele Romagnoli ${ }^{1}$, Daniele D'Agostino ${ }^{1}$, Antonio Salvaggio ${ }^{1}$, \\ Marco Giampaoli ${ }^{1}$, Paolo Corsi ${ }^{1}$, Lorenzo Bianchi ${ }^{1}$, Marco Borghesi ${ }^{2}$, Riccardo Schiavina ${ }^{2}$, \\ Eugenio Brunocilla ${ }^{2}$, Peter Wiklund ${ }^{3}$, Angelo Porreca ${ }^{1}$ \\ ${ }^{1}$ Department of Urology, Policlinic of Abano Terme (Padova), Italy; \\ ${ }^{2}$ Department of Urology, S. Orsola-Malpighi Hospital, University of Bologna, Italy; \\ ${ }^{3}$ Department of Urology, Mount Sinai Institution, Icahn School of Medicine, USA.
}

\begin{abstract}
Summary Objective: The aim of our study is to describe the use of posterior muscle-fascial reconstruction during urethro-ileal anastomosis in bladder cancer (BC) patients submitted to robot-assisted radical cystectomy $(R C)$ with orthotopic neobladder $(\mathrm{ON})$ and its role in facilitating day- and night-time continence recovery during a 12-month follow up. Materials and methods: We prospectively collected data from 42 consecutive patients who underwent RARC with totally intracorporeal ON and extended pelvic lymph node dissection (PLND) at our Institution from June 2014 to October 2017.

Prior to the urethro-neobladder anastomosis we reconstructed the Denonvilliers Fascia (DF) as previously described for radical prostatectomy using a bidirectional barbed suture. Day and night-time recovery rates were reported at 3, 6 and 12 months after surgery, with continent patients being those using either no urinary pads or 1 safety pads.

Results: Median age at surgery was 63 yrs, 41 (97.6\%) patients were male. $28(66.7 \%)$ patients presented a clinical T2 disease. Median operative time and median $\mathrm{ON}$ reconstruction time were 450 minutes and 180 minutes respectively. 13 (31\%) individuals had non-organ confined disease, with 11 (26.2\%) patients with positive lymph nodes (median 3 positive lymph nodes) and 2 (4.8\%) with non-urothelial cancer at final pathologic examination. Median hospital stay and median catheterization time were 7 (IQR 7-8) and 21 (IQR 19-22). During first 30 post-operative days we recorded 7 (16.7\%) low-grade Clavien and 2 (4.8\%) III C Clavien complications, whereas between 30 and 90 postoperative days we recorded 4 (9.5\%) low-grade, 4 (9.5) III a and 1 (2.4\%) IIIb complications. Day-time and night-time continence rates were $61.9 \%$ vs $52.4 \%$, $73.8 \%$ vs $64.3 \%$ and $90.5 \%$ vs $73.8 \%$ at three, six and twelve months follow up. Day-time continence was significantly superior in the younger group (97\% vs $57 \%$, p 0.01); night-time continence rates were also superior among $<70$ yrs patients, despite not reaching statistical significance ( $77 \%$ vs $57 \%$, p 0.3).

Conclusions: Posterior muscle-fascial reconstruction aids continence recovery in $B C$ patients undergoing $R A R C$ with $O N$, with younger and fitter patients most benefitting from ON reconstruction.
\end{abstract}

KEY WORDS: RARC; Orthotopic neobladder; Posterior musclefascial reconstruction; Robotic surgery; Radical cystectomy.

Submitted 8 January 2019; Accepted February 2019

\section{INTRODUCTION}

Radical cystectomy with pelvic lymph node dissection represents the gold standard treatment for muscle-invasive bladder cancer (1). Despite ileal conduit (IC) being the most commonly performed type of urinary derivation (UD), orthotopic neobladder (ON) reconstruction might lead to a better quality of life, mainly due to a better preservation of patient's self-image, especially among younger individuals (2). Robot-assisted radical cystectomy (RARC) was first described more than 10 years ago and is steadily gaining popularity among tertiary care centers, but most cases of ON are performed through a minilaparotomy $(3,4)$. Totally intracorporeal robot-assisted ON have been shown to be comparable in terms of urodynamic profiles to open $\mathrm{ON}$, as high-volume and lowpressure reservoirs, despite worse continence rates during the first months after surgery (5). Continence rate usually improve throughout follow up, as the $\mathrm{ON}$ requires months to reach its full functional capacity, with $75-95 \%$ and $50-85 \%$ day-time and night-time continence rates at long term follow-up, respectively (6-8). The aim of our study is to describe our novel technique to perform urethra-neobladder anastomosis and to present its functional results through a 12 months follow-up, with particular regard to day-time and night-time continence rates.

\section{MATERIALS AND MethOdS}

We prospectively collected data from 42 patients who consecutively underwent RARC with pelvic lymph node dissection (PLND) and totally intracorporeal orthotopic neobladder (ON) reconstruction from June 2014 to October 2017 at our Institution. Every surgical procedure was performed by 2 surgeons (AP and AS) with previous experience of urologic robot-assisted surgery. The first procedures were performed after completing a modular training program under a skilled surgeon (PW) (9). Surgeons from a second Tertiary Center participated as table-assistants, after a week of video sessions, as a part of a structured modular training to gradually master 
the technique. For each patient we prospectively reported intra- and peri-operative data, with complete pathologic data. Complications were stratified as early, during first 30 post-operative days, and late, from 30 to 90 postoperative days, and were graded according to ClavienDindo classification. Day-time and night-time continence were evaluated at 3,6 and 12-month follow up. Continence was defined as the need for 1 safety pad or urine loss inferior than $10 \mathrm{~g}$ at pad test (10).

\section{Indications}

RARC with ON was proposed to patients with either muscle-invasive bladder cancer (MIBC) or non-muscle-invasive bladder cancer (NMIBC) with high risk features, namely high-grade tumour with carcinoma in situ (CIS), recurrent high-grade disease after bladder instillations with Bacillus Calmette-Guerin (BCG), multiple and/or large high-grade tumours, recurrent multifocal large low-grade tumours $(11,12)$. Previous abdominal surgery wasn't considered as an absolute contraindication for robotic surgery, albeit it could lead to sometimes long pre-operative laparoscopic lysis of peritoneal adherences. The presence of tumour of the prostatic urethra leads to a higher chance of urethral lesions, although it is not considered as an absolute contraindication for $\mathrm{ON}$ reconstruction (13). An intra-operative frozen section was performed to exclude tumour localization of the urethral stump, despite missing 33\% of CIS at this site (14). As commonly scheduled to prostate cancer patients undergoing radical prostatectomy (RP), pre-operative mpMRI was performed to determine the extent of nervesparing (NS) surgical plan in younger and sexually active individuals (15). Compromised renal and/or hepatic function were considered as excluding factors for $\mathrm{ON}$ reconstruction, as well as severe chronic bowel inflammation. Age and previous abdominal radiotherapy were not considered as absolute contraindications, although elderly patients share a higher risk of enuresis and nighttime incontinence $(8,16-18)$.

\section{Surgical procedure}

With the patient in steep Trendelenburg position we perform trans-peritoneal approach using $\mathrm{Da}$ Vinci $X i^{\circledR}$ Surgical System (Intuitive Surgical, Sunnyvale, CA, USA). The ureters are isolated from $4-5 \mathrm{~cm}$ below the pelvicureteral junction to the Waldeyer's sheath, where they are clipped using 2 Hemo-Locks ${ }^{\circledR}$. The left ureter is transposed through the sigmoid mesocolon. The radical cystoprostatectomy with extended pelvic lymph node dissection is performed as previously described $(19,20)$. For the only female patient included in our study the uterus and the ovaries were removed along with the anterior wall of the vagina, whose identification was eased by manipulating a sponge in the vagina. After proper examination, no signs of tumour invasion of the vaginal walls were detected, thus allowing us to also preserve the autonomic nerves that run through its lateral walls. The vaginal edges were finally closed using a "clam-shell technique" (20).

Uni- or bilateral nerve-sparing was performed whenever oncologically safe, according to pre-operative mpMRI results. During the initial part of radical cystectomy, the peritoneum was incised just below the tips of the seminal vesicles to expose the Denonvilliers' fascia (DF). In case of nerve-sparing procedures the fascia was then opened and a surgical plane between DF and the rectum was developed uni or bi-laterally, as performed during robotic prostatectomy (21).

It is essential to spare as much as possible the rhabdosphincter as well as distal urethra, to maintain an adequate closure pressure. After dissection of the prostatic apex, a frozen section of the distal urethra is performed to exclude urethral tumours. Each patient also underwent extended PLND, including external as well as internal and common iliac lymph nodes (LN), obturator fossa and pre-sacral area LN. After removal, the cystoprostatectomy specimen and LN are placed in two different impermeable bags to avoid leakage of neoplastic cells. The Da Vinci $X i^{\circledR}$ is then un-docked, and patient position is flattened to around $10-15^{\circ}$ of Trendelenburg to proceed with the reconstructive phase.

\section{Anastomosis technique and totally intracorporeal neobladder reconstruction}

After further docking of Da Vinci Xi ${ }^{\circledR}$ Surgical System, a distal ileal segment is mobilized to reach down the urethral stump. Using a 35 or $45 \mathrm{~cm}$ bidirectional barbed suture 3/0 (Filbloc ${ }^{\circledR}$ Assut, Europe) as a first step we stitch the Denonvilliers Fascia (DF) with both needles, as performed during urethro-vesical anastomosis of radical prostatectomy (RP) (21-23). Using the left needles, the DF is gently transposed to the fibrous part of the sphincter with to bites from left to right. The DF is then firmly attached to the fibrous sphincter using the right end of the suture. In our opinion, this step is as important during $\mathrm{ON}$ as for RP. The distribution of tensions will reduce the tractions upon the urethro-ileal anastomosis, limiting urinary leakages and facilitating urinary continence recovery. A $20 \mathrm{~F}$ opening is then created on the anti-mesenteric edge of the lower ileal segment using robotic scissors. During the second step, the left end is used to approximate the posterior side of urethral stump to the lower margin of the $20 \mathrm{~F}$ opening of the ileal segment using two bites from left to right. With the right end the posterior distal urethra is sutured to the lower margin of the ileal opening. During the third step the urethro-ileal anastomosis is then completed stitching the anastomosis anticlockwise from 5' to 12' using the right end and clockwise from 7' to 12' using the left end according to Van Velthoven technique. A 20 F tri-lumen catheter is then placed through the anastomosis. The ON is then completed according to the technique described by Hosseini using a $50 \mathrm{~cm}$ distal ileal segment with a full intracorporeal approach (20).

\section{Statistical analysis}

Continuous variables were reported as medians with interquartile ranges (IQR) and categorical variables were described as frequencies with percentages. Chi-square was used to compare day- and night-time urinary recovery rates among patients aged $<70$ yrs and those $\geq 70$ yrs, using a one-tailed 95\% confidence interval. Statistical analysis was performed with Statistical Package for Social Science (SPSS) v 21 for Macintosh. 


\section{RESULTS}

Table 1 depicts pre-operative features. 41 out of 42 patients were male, with median age of 63 yrs, median American Society of Anaesthesiology (ASA) score of 2 and median BMI of 26.

28 (66.7\%) had MIBC with 11 individuals (26.2\%) with associated CIS; 39 BC patients (92.9\%) had a high-grade disease, 15 (35.7\%) were submitted to intravesical instillations of BCG and 14 (33.3\%) underwent a cycle of adjuvant chemotherapy. Median operative time was $450 \mathrm{~min}-$ utes (Table 2), with median cystectomy time of 120 minutes, median PLND time of 90 minutes and median ON reconstruction time of 180 minutes. Median estimated blood loss (EBL) was $225 \mathrm{ml}, 3$ (7.1\%) patients received intra-operative blood transfusions, 17 (41.5\%) and 7 (17.1\%) underwent bilateral and unilateral NS preservation, respectively. No patient was converted to laparotomic cystectomy. 2 (4.8\%) patients had CIS at final pathology, 8 (19\%) had T0 disease and 13 (31\%) had non-organconfined BC; 9 (21.4\%) patients had concomitant CIS, 1 (2.4\%) had positive BC margins and 2 (4.8\%) individuals presented a squamous cell carcinoma (SCC). Each patient underwent extended PLND, with a median of 31 lymph node (LN) retrieved. 11 patients had positive lymph nodes at final pathology, with a median of 3 (IQR 1-9) LN involved. 17 (41.4\%) patients had concomitant prostatic carcinoma (PC), with 12 (29.3\%) Gleason grade 1 and 5 (12.2\%) grade 2 disease, respectively, and just one case (2.4\%) of positive surgical margins for PC.

Post-operative data are shown in Table 3. Median hospital stay was 7 days (IQR 7-8). Median catheterization time was 21 days (IQR 19-22). During first 30 postoperative days we recorded 7 (16.7\%) low Clavien grade complications and 2 (4.8\%) grade IIIa complications,

Table 1.

Patients' demographic and pre-operative data.

\begin{tabular}{|lc|}
\hline Number of patients (\%) & $\mathbf{4 2}(\mathbf{1 0 0 )}$ \\
\hline Sex (\%) & $41(97.6)$ \\
Male & $1(2.4)$ \\
Female & 63 \\
\hline Age at surgery & $(59-68)$ \\
Median & \\
IQR & 2 \\
\hline ASA score & $22-3$ \\
Median & 26 \\
IQR & $23.6-28$ \\
\hline BMI & $3(7.1)$ \\
Median & $(7.1)$ \\
IQR & $8(19)$ \\
\hline Pre-operative stage & $28(66.7)$ \\
CIS & $0(0)$ \\
Ta3 & \\
T1 & $3(7.1)$ \\
T2 & $39(92.9)$ \\
T3-T4 & $11(26.2)$ \\
\hline Pre-operative grade & $15(35.7)$ \\
Low grade G1-G2 (\%) & $14(33.3)$ \\
Highgrade G3 (\%) & \\
\hline Concomitant CIS (\%) & \\
Previous BCG instillation & \\
Neo-adjuvant CHT (\%) & \\
\hline IQR: Interquartile range; ASA: American Society of Anesthesiology; BMl: & Body mass index; \\
CIS: Carcinoma in situ; BCG: Bacillus Calmette-Guérin; CHT: Chemotherapy. \\
\hline
\end{tabular}

Table 2.

Patients' peri-operative data.

\begin{tabular}{|c|c|}
\hline $\begin{array}{l}\text { Total operative time (min) } \\
\text { Median } \\
\text { IQR }\end{array}$ & $\begin{array}{c}450 \\
410-480\end{array}$ \\
\hline \multicolumn{2}{|l|}{ Cystectomy time (min) } \\
\hline $\begin{array}{l}\text { Median } \\
\text { IQR }\end{array}$ & $\begin{array}{c}120 \\
80-150\end{array}$ \\
\hline \multicolumn{2}{|l|}{ PLND time (min) } \\
\hline $\begin{array}{l}\text { Median } \\
\text { IQR }\end{array}$ & $\begin{array}{c}90 \\
80-110\end{array}$ \\
\hline \multicolumn{2}{|l|}{$\overline{\text { ON time (min) }}$} \\
\hline $\begin{array}{l}\text { Median } \\
\text { IQR }\end{array}$ & $\begin{array}{c}180 \\
120-240\end{array}$ \\
\hline \multicolumn{2}{|l|}{ Estimated blood loss (ml) } \\
\hline $\begin{array}{l}\text { Median } \\
\text { IQR }\end{array}$ & $\begin{array}{c}225 \\
127.5-312.5\end{array}$ \\
\hline Intraoperative blood transfusions (\%) & $3(7.1)$ \\
\hline Conversion to open surgery (\%) & $0(0)$ \\
\hline \multicolumn{2}{|l|}{ NS procedure (\%)* } \\
\hline No NS & $17(41.5)$ \\
\hline Unilateral NS & 7 (17.1) \\
\hline Bilateral NS & $17(41.5)$ \\
\hline \multicolumn{2}{|l|}{ Pathologic stage (\%) } \\
\hline $\mathrm{CIS}$ & $2(4.8)$ \\
\hline TO & $8(19)$ \\
\hline $\mathrm{T} 1$ & $9(21.4)$ \\
\hline $\mathrm{T} 2$ & $10(23.8)$ \\
\hline T3 & $9(21.4)$ \\
\hline T4 & $4(9.5)$ \\
\hline Concomitant CIS (\%) & $9(21.4)$ \\
\hline Positive surgical margins (\%) & $1(2.4)$ \\
\hline \multicolumn{2}{|l|}{ Hystotype (\%) } \\
\hline No tumor & $8(19)$ \\
\hline TCC & $32(76.2)$ \\
\hline SCC & $2(4.8)$ \\
\hline PLND (\%) & $42(100)$ \\
\hline \multicolumn{2}{|l|}{ Number of $L N$ retrieved } \\
\hline Median & 31 \\
\hline IQR & $24-37$ \\
\hline$\overline{\mathrm{N}+(\%)}$ & $11(26.2)$ \\
\hline \multicolumn{2}{|l|}{ Number of positive LN \# } \\
\hline Median & 3 \\
\hline IQR & $1-9$ \\
\hline Incidental PCa (\%)* & $17(41.4)$ \\
\hline \multicolumn{2}{|l|}{ Gleason grade group* } \\
\hline 1 & $12(29.3)$ \\
\hline 2 & $5(12.2)$ \\
\hline $3-5$ & $0(0.0)$ \\
\hline$\overline{\text { Positive PCa margins (\%) } * *}$ & $1(6 \%)$ \\
\hline \multicolumn{2}{|c|}{$\begin{array}{l}\text { IQR: Interquartile range; PLND: Pelvic lymph node dissection; ON: Orthotopic neobladde } \\
\text { NS: Nerve-sparing; CIS: Carcinoma in situ; TCC: Transitional cell carcinoma; } \\
\text { SCC: Squamous cell carcinoma; LN: Lymph node; PCa: Prostate cancer. } \\
\text { ^ Among male patients; *^ Among male patients with concurrent PCa; } \\
\text { \# Patients with positive lymph nodesy. }\end{array}$} \\
\hline
\end{tabular}

with $4(9.5 \%)$ individuals who were re-admitted at our Institution. Between 30 and 90 post-operative days 4 (9.5\%) low grade Clavien complications were registered, with 4 (9.5\%) grade IIIa complications and 1 (2.4\%) IIIb complication (due to urinary leakage from the $\mathrm{ON}$ ), with a total of 7 patients re-admitted at our Institution. No patients deceased during first 90 post-operative days. 22 (55.3\%) patients reported regular sexual intercourses after 90 post-operative days with oral phosphodiesterase-5 inhibitors (PDE 5). 
Table 3.

Post-operative and within-90 post operative days features.

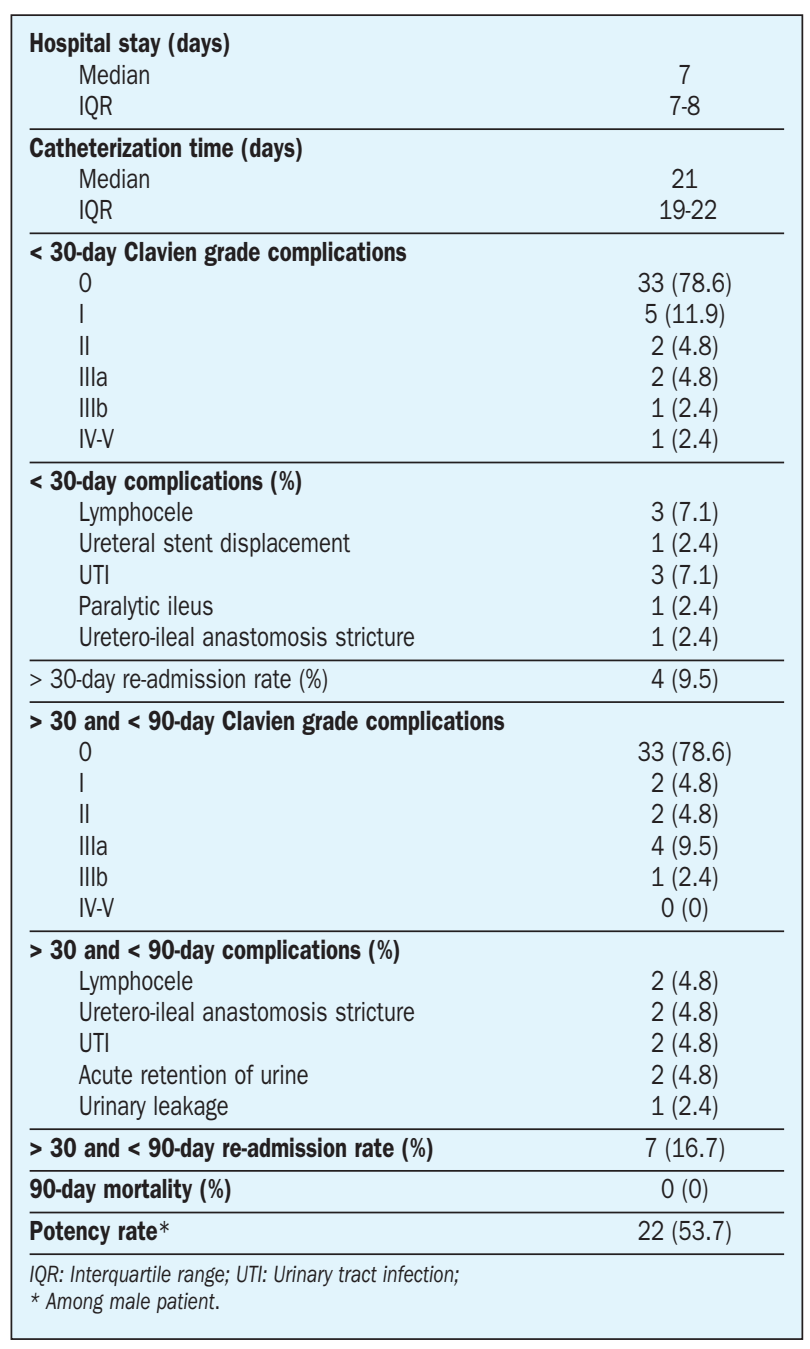

\section{Continence recovery}

Table 4 a depicts overall urinary recovery rate, with $61.9 \%, 73.8 \%$ and $90.5 \%$ continent patients during daytime at 3, 6 and 12 months, respectively; night-time continent patients also increased during our 12-month follow-up, with 22 (52.4\%), 27 (64.3\%) and 31 (73.8\%)

Table $4 a$.

Overall day- and night-time continence rate at 3, 6 and 12month follow up.

\begin{tabular}{|lccc|}
\hline & $\mathbf{3}$ months & $\mathbf{6}$ months & $\mathbf{1 2}$ months \\
\hline Day-time continence & $26(61.9)$ & $31(73.8)$ & $38(90.5)$ \\
\hline Night-time continence & $22(52.4)$ & $27(64.3)$ & $31(73.8)$ \\
\hline
\end{tabular}

Table 4b.

Day- and night-time continence rates at 12 months stratified according to age at surgery.

\begin{tabular}{|lccc|}
\hline & $<\mathbf{7 0}$ yrs & $\geq \mathbf{7 0}$ yrs & p-value \\
\hline Day-time continence & $34(97.2)$ & $4(57.1)$ & 0.01 \\
\hline Night-time continence & $27(77.1)$ & $4(57.1)$ & 0.3 \\
\hline
\end{tabular}

continent patients at 3, 6 and 12 months as the ON gradually reached its full functional capacity.

After stratifying 12-month continence rates according to age at surgery (namely $<70$ yrs vs $\geq 70$ yrs, Table $4 b$ ) daytime continence was significantly superior in the younger group (97\% vs 57\%, p 0.01); night-time continence rates were also superior among $<70$ yrs patients, despite not reaching statistical significance ( $77 \%$ vs $57 \%$, p 0.3 ).

\section{Discussion}

In the past decade, many surgeons tried to translate different types of ON, formerly proposed for open radical cystectomy (ORC), for RARC, in order to simplify technique, thus reducing operative times, and to obtain better functional outcomes. The lack of tactile feedback demands a careful manipulation of the bowel down to the deep pelvis, with steep Trendelenburg position. No cases of colonic ON have been described with robotic surgery; each reported technique describe a refluxing uretero-ileal anastomosis (3). Pruthi et al. described a novel technique for robotic neobladder, a U-shaped reservoir without bowel cross-folding and using a stapler device to reduce intraoperative time (24). As evidenced by other authors, also in our series $\mathrm{ON}$ reconstruction is the most time-consuming step during RARC, with a median time of 180 minutes, compared to 120 minutes for radical cystectomy and 90 minutes for extended PLND. Although operative times should lower by gaining more experience with this procedure, this phase is indeed a limiting step for a widespread diffusion of this technique. The lack of cross-folding and the use of a stapler device could however translate in a quicker renal impairment due to higher reservoir pressures and a higher rate of neobladder calculi respectively. As recently evidenced by RAZOR randomized controlled trial, RARC is not related to fewer early and late post-operative complications than ORC. Despite longer operative times than ORC, RARC is related to a lower EBL, lower blood transfusion rate and shorter hospital stay, with comparable oncologic outcomes (25).

Previously, Bochner and al. reported similar outcomes in terms of comorbidities and mortality between ORC and RARC with extracorporeal ON, with higher costs related to robotic surgery (26). During first 90 post-operative days, we recorded 11 Clavien low-grade complications and 7 grade III complication, of those only one was submitted to a further surgical procedure under general anesthesia to repair a urinary fistula. Ureteral strictures represent the most frequent long-term complications after RC with different types of UD, with subsequent loss of renal functions, upper urinary tract infections and need for further invasive procedures. The exact cause for ureteral strictures is unclear but anastomotic ischemia has been proposed as the main factor contributing to their relatively high incidence. (27) In our series 3 (7\%) patients developed ureteral strictures during our 3-month follow up, with available literature reports of $5 \%$ to $10 \%$ of ureteral strictures after RC with uretero-ileal anastomosis; preoperatively dilated ureters are more likely to develop strictures months after surgery $(28,29)$.

The management of ureteral strictures is mostly endoscopic and laparoscopic/robot-assisted among Tertiary 
Care centers, thus reserving laparotomic surgery to a small number of patients (30).

All-grade Clavien complication rate was $48.9 \%$, which is slightly lower than reported $>60 \%$, with up to $7 \%$ of mortality in some series, which could proof a good patient selection for $\mathrm{ON}$ reconstruction, as further confirmed by a median hospital stay of 7 days. Moreover, median EBL and intraoperative transfusion rates were exceptionally lower than those reported by other authors for totally intracorporeal $\mathrm{ON}(31,32)$.

The choice of ON has to be carefully discussed with the patient, with many variables such as age, pre-operative cognitive function, general health, comorbidities, disease stage, post-operative rehabilitation, that are needed to be taken into account when deciding the most suitable type of UD $(33,34)$. Indeed, younger and fitter patients with low tumour burden are the most likely to benefit from $\mathrm{ON}$ reconstruction, with better continence rates and higher QoL scores during follow-up (2). Urethro-ileal anastomosis is a crucial step during $\mathrm{ON}$ reconstruction. A tension-free anastomosis is paramount to prevent strictures and urinary leakages from the urethro-ileal anastomosis. A proper preservation of the external urinary sphincter when dissecting the prostatic apex and the identification of an ileal segment with an adequately long mesentery are crucial to reduce as possible tension when suturing $\mathrm{ON}$ to the membranous urethra (3). The length of membranous urethra has been itself identified as a very important parameter to predict good continence recovery rates after RP (35). In this context, posterior musculofascial reconstruction with subsequent urethro-ON anastomosis using a bidirectional barbed suture, as described for RP, limits tension upon the anastomosis, thus reducing urinary leakages and acute urinary retentions related to strictures $(21,22,36)$. In our series, despite a relatively short follow-up, we registered only one case of acute urinary retention and only one urinary leakage, which needed a further surgical procedure to repair the fistula. Continence rates at 12 month-follow up were in line with those previously reported for ORC of $75-95 \%$ and 50 $85 \%$ continence rates during day-time and night-time, respectively (8). After stratifying 12-month recovery rates according to age at surgery, day-time and night-time continence rates were superior for patients aged $<70$ yrs, despite night-time continence couldn't reach statistical significance due to the low number of elderly patients. Many authors indicated urinary leakage to be the main culprit of lower QoL scores for patients submitted to ON versus those with $\mathrm{IC}(37,38)$. A complete recovery of urinary continence after radical cystectomy with $\mathrm{ON}$ is deeply connected to the full maturation of the newly created reservoir. The estimated time to reach a 300-500 ml is around 8 months. Elderly patients are more likely to experience incontinence due to apoptosis of rhabdosphincter cells, with reduced urethral closure pressure, lower sphincter length and progressive denervation of the membranous urethra. Furthermore, nerve-sparing techniques have been found to improve continence rates in patients with $\mathrm{ON}(6,7,39)$. According to the epidemiological studies $5 \%$ of individuals newly diagnosed with $\mathrm{PCa}$ declared incontinence prior to any surgical procedure regardless of age, with older individuals with other urinary disturbances reporting even pre-operative higher incontinence rates (40). Hence, an accurate assessment of urinary continence in patients undergoing $\mathrm{RC}$ with $\mathrm{ON}$ should be explored, as incontinent patients wouldn't benefit from an orthotopic reconstruction. At last, potency rates were exceptionally good, as 22 out of 24 male patients who underwent uni- or bilateral nerve sparing procedures regular sexual intercourses w/o oral medications with PDE5-I, which further confirms a proper patient selection for this kind of procedure. The main limitations of this study are the relatively small cohort of patient, the lack of a control group, a relatively short follow-up especially for long-term complications and perhaps the lack of urodynamic studies.

\section{Conclusions}

Posterior musclefascial reconstruction before performing urethro-ileal anastomosis helps improving urinary continence in patients undergoing RARC with ON, with comparable results to ORC. A proper patient selection is paramount to fully benefit from $\mathrm{ON}$, with younger and fitter patients experiencing better continence and potency rates. Further studies will be needed to assess whether a posterior reconstruction could translate in changes of the urodynamic profile.

\section{REFERENCES}

1. Stein JP1, Lieskovsky G, Cote R, et al. Radical cystectomy in the treatment of invasive bladder cancer: long-term results in 1,054 patients. J Clin Oncol 2001; 19:666-75.

2. Dutta SC, Chang SC, Coffey CS, et al. Health related quality of life assessment after radical cystectomy: comparison of ileal conduit with continent orthotopic neobladder. J Urol 2002; 168:164-7.

3. Tan WS, LambBW, Kelly JD. Evolution of the neobladder: A critical review of open and intracorporeal neobladder reconstruction techniques. Scand J Urol. 2016; 50:95-103.

4. Ahmed K, Khan SA, Hayn MH. Analysis of intracorporeal compared with extracorporeal urinary diversion after robot-assisted radical cystectomy: results from the International Robotic Cystectomy Consortium. Eur Urol. 2014; 65:340-7.

5. Satkunasivam R, Santomauro M, Chopra S, et al. Robotic Intracorporeal Orthotopic Neobladder: Urodynamic Outcomes, Urinary Function, and Health-relted Quality of Life. Eur Urol. 2016; 69:247-53.

6. Madersbacher S, Möhrle K, Burkhard F, Studer UE. Long-term voiding pattern of patients with ileal orthotopic bladder substitutes. J Urol. 2002; 167:2052-7.

7. Strasser H, Tiefenthaler M, Steinlechner M, et al. Urinary incontinence in the elderly and age-dependent apoptosis of rhabdosphincter cells. Lancet. 1999; 354:918-9.

8. Minervini A, Serni S, Vittori G, et al. Current indications and results of orthotopic ileal neobladder for bladder cancer. Expert Rev Anticancer Ther. 2014; 14:419-30.

9. Porreca A, Chessa F, Romagnoli D, et al. Robot assisted radical cystectomy with totally intracorporeal urinary diversion: initial, single-surgeon's experience after a modified modular training. Minerva Urol Nefrol. 2018; 70:193-201.

10. Kretschmer A, Grimm T, Buchner A, et al. Prognostic features for objectively defined urinary continence after radical cystectomy 
and ileal orthotopic neobladder in a contemporary cohort. J Urol. 2017; 197:210-215.

11. Sylvester RJ, van der Meijden AP, Oosterlinck W, et al. Predicting recurrence and progression in individual patients with stage Ta T1 bladder cancer using EORTC risk tables: a combined analysis of 2596 patients from seven EORTC trials. Eur Urol. 2006; 49:466-5.

12. Fernandez-Gomez J, Madero R, Solsona E, et al. Predicting nonmuscle invasive bladder cancer recurrence and progression in patients treated with bacillus Calmette-Guerin: the CUETO scoring model. J Urol. 2009; 182:2195-203.

13. Autmann RE, Abol-Enein H, Davidsson T, et al. ICUD-EAU International Consultation on Bladder Cancer 2012: Urinary diversion. Eur Urol. 2013; 63:67-80.

14. Gordetsky J, Bivalacqua T, Schoenberg M, Epstein JI. Ureteral and urethral frozen sections during radical cystectomy or cystoprostatectomy: an analysis of denudation and atypia. Urology. 2014; 84:619-23.

15. Schiavina R, Bianchi L, Borghesi M, et al. MRI Displays the prostatic cancer anatomy and improves the bundles management before robot-assisted radical prostatectomy. J Endourol. 2018; 32:315-321.

16. Studer UE, Hautmann RE, Hohenfellner M, et al. Indications for continent diversion after cystectomy and factors affecting long-term results. Urol Oncol. 1998; 4:172-82.

17. Mills RD, Studer UE, Metabolic consequences of continent urinary diversion. J Urol. 1999; 161:1057-66.

18. Skinner DG, Studer UE, Okada K, et al. Which patients are suitable for continent diversion or bladder substitution following cystectomy or other definitive local treatment? Int J Urol. 1995; 2 (Suppl 2):105-12.

19. Desai MM, Berger AK, Brandina RR, et al. Robotic and laparoscopic high extended pelvic lymph node dissection during radical cystectomy: technique and outcomes. Eur Urol. 2012; 61:350-5.

20. Hosseini A, Adding C, Nilsson A, et al. Robotic cystectomy: surgical technique. BJU Int. 2011; 108:962-8.

21. Porreca A, D'Agostino D, Dandrea M, et al. Bidirectional barbed suture for posterior musculofascial reconstruction and knotless vesicourethral anastomosis during robot-assisted radical prostatectomy. Minerva Urol Nefrol. 2018; 70:319-325.

22. Ficarra V1, Gan M, Borghesi M, et al. Posterior muscolofascial reconstruction incorporated into urethrovescical anastomosis during robot-assisted radical prostatectomy. J Endourol. 2012; 26:1542-5.

23. Lin YF, Lai SK, Liu QY, et al. Efficacy and safety of barbed suture in minimally invasive radical prostatectomy: A systematic review and meta-analysis. Kaohsiung J Med Sci. 2017; 33:107-115.

24. Pruthi RS, Nix J, McRackan D, et al. Robotic-assisted laparoscopic intracorporeal urinary diversion. Eur Urol. 2010; 57:1013-21.

25. Parekh DJ, Reis IM, Castle EP, et al. Robot-assisted radical cystectomy versus open radical cystectomy in patients with bladder cancer (RAZOR): an open-label, randomised, phase 3, non-inferiority trial. Lancet. 2018; 391:2525-2536.

26. Bochner BH, Dalbagni G, Sjoberg DD, et al. Comparing open radical cystectomy and robot-assisted laparoscopic radical cystectomy: a randomized clinical trial. Eur Urol. 2015; 67:1042-1050.

27. Lobo N, Dupré S, Sahai A, et al. Getting out of a tight spot: an overview of ureteroenteric anastomotic strictures. Nat Rev Urol. 2016; 13:447-55.

28. Hosseini A, Dey L, Laurin O, et al. Ureteric stricture rates and management after robot-assisted radical cystectomy: a single-centre observational study. Scand J Urol. 2018; 52:244-248.
29. Hautmann RE, de Petriconi $R$, Kahlmeyer $A$, et al. Preoperatively dilated ureters are a specific risk factor for the development of ureteroenteric strictures after open radical cystectomy and ileal neobladder. J Urol. 2017; 198:1098-1106.

30. Schiavina R, Zaramella S, Chessa F, et al. Laparoscopic and robotic ureteral stenosis repair: a multi-institutional experience with a long-term follow-up. J Robot Surg. 2016; 10:323-330.

31. Novara G, Catto JW, Wilson T, et al. Systematic review and cumulative analysis of perioperative outcomes and complications after robotassisted radical cystectomy. Eur Urol. 2015; 67:376-401.

32. Moeen AM, Safwat AS, Elderwy AA, et al. Management of neobladder complications: endoscopy comes first. Scand J Urol. 2017; 51:146-151.

33. Ali AS, Hayes MC, Birch B, et al. Health related quality of life (HRQoL) after cystectomy: comparison between orthotopic neobladder and ileal conduit diversion. Eur J Surg Oncol. 2015; 41:295-9.

34. Månsson A, Davidsson T, Hunt S, Månsson W. The quality of life in men after radical cystectomy with a continent cutaneous diversion or orthotopic bladder substitution: is there a difference? BJU Int. 2002; 90:386-90.

35. Paparel P, Akin O, Sandhu JS, et al. Recovery of urinary continence after radical prostatectomy: association with urethral length and urethral fibrosis measured by preoperative and postoperative endorectal magnetic resonance imaging. Eur Urol. 2009; 55:629-37.

36. Porreca A, Salvaggio A, Dandrea M, et al. Robotic-assisted radical prostatectomy with the use of barbed sutures. Surg Technol Int. 2017; 30: p. 39-43.

37. Gilbert SM, Wood DP, Dunn RL, et al. Measuring health-related quality of life outcomes in bladder cancer patients using the Bladder Cancer Index (BCI). Cancer, 2007; 109:1756-62.

38. Hedgepeth RC, Gilbert SM, He C, et al. Body image and bladder cancer specific quality of life in patients with ileal conduit and neobladder urinary diversions. Urology. 2010; 76: p. 671-5.

39. Gilpin SA, Gilpin CJ, Dixon JS, et al. The effect of age on the autonomic innervation of the urinary bladder. Br J Urol. 1986; 58:378-81

40. Porreca A, Noale $M$, Artibani W, et al. Pros-IT CNR study group. Disease-specific and general health-related quality of life in newly diagnosed prostate cancer patients: the Pros-IT CNR study. Health Qual Life Outcomes, 2018; 16:122.

\section{Correspondence}

Federico Mineo Bianchi, MD (Corresponding Author) federico.mineobianchi@gmail.com Lorenzo Bianchi, MD - lorenzo.bianchi3@gmail.com Marco Borghesi, MD - mark.borghesil@gmail.com Riccardo Schiavina, MD - rschiavina@yahoo.it Eugenio Brunocilla, MD - eugenio.brunocilla@unibo.it S. Orsola-Malpighi Hospital, University of Bologna, Via Palagi 9, Bologna (Italy)

Daniele Romagnoli, MD - danieleromagnoli87@gmail.com Daniele D’Agostino, MD - dott.dagostino@gmail.com Antonio Salvaggio, MD - asalvaggio@casacura.it

Marco Giampaoli, MD - giampaoli.marco85@gmail.com Paolo Corsi, MD - pcorsi@casacura.it

Angelo Porreca, MD - angeloporreca@gmail.com

Policlinic of Abano Terme,

Piazza Cristoforo Colombo 1, Abano Terme, Padua (Italy)

PeterWiklund, MD - peter.wiklund@mountsinai.org Mount Sinai, 625 Madison Avenue, New York City, New York (USA) 